\title{
Estratégias de comercialização da soja: análise de portfólios, sob condições de risco
}

\author{
Judas Tadeu Grassi Mendes IBMEC \\ João Batista Padilha Junior Universidade Federal do Paraná
}

\section{RESUMO}

A soja é a cultura mais importante do agronegócio brasileiro. Entretanto, na época da comercialização, os sojicultores defrontam-se com o dilema de "quanto", "quando" e "como" vender sua produção, devido ao fato de a mesma ser uma atividade realizada sob condições de risco e de incerteza. Através da utilização de um modelo matemático de programação quadrática, buscou-se determinar os melhores portfólios, no sentido de maximizar a renda com certo nível de risco, ou minimizar o risco para certos níveis de renda. O portfólio mais eficiente obtido entre todos diz respeito a produtores com "média aversão ao risco" $(\alpha=0,30)$. Dessa forma, tal plano ótimo de comercialização indica que os produtores de soja do Estado do Paraná poderiam armazenar a produção na época da colheita com posterior venda mensal fracionada, o que representaria um total de $90,8 \%$ das vendas concentrando-se nos últimos quatro meses do ano.

PALAVRAS-CHAVE

Soja, risco, portfólios, programação quadrática.

\section{Soybeans marketing strategies: portfolio analysis; under risk conditions}

\begin{abstract}
The soybeans complex represents a fundamental role in the brazilian agribusiness. However, at the time of soybeans marketing, producers face a dilemma: "when", "how much" and "how" to sell their production, due to the uncertainties of this activity under risk conditions. By this way, using a mathematical model of quadratic programming, this work has searched for the best portfolio, trying to increase their incomes with some level of risk or to decrease risks at some income level. The most efficient plan is related to producers with medium risk aversion $(\alpha=0,3)$. This optimum marketing plan shows that soybean producers of Paraná State could store production at the harvest time in order to sell later following, for instance, this schedule: 21,9\% in September; 25, 1\% in October; 19,9\% in November and 23,9\% in December, which means that 90,8\% of all sales concentrated on the last four months of the year.
\end{abstract}

\section{KEY WORDS}

Soybeans, risk, portfolio, quadratic programming. 


\section{INTRODUÇÃO}

Não há nenhum produto agrícola no Brasil que tenha experimentado uma expansão de área tão extraordinária quanto a soja, que de uma produção inferior a 1 milhão de toneladas ao final dos anos 60 já ultrapassa 50 milhões de toneladas em 2008. Com uma área cultivada em torno de 20 milhões de hectares, pode ser considerada a atividade agrícola de maior expressão no país. Atualmente, a área cultivada com soja no Brasil representa quase $40 \%$ da área de grãos no país. A partir dos anos 1980, depois de consolidado o plantio nos três estados do Sul (RS, SC e PR), a soja passou a se expandir em outros estados, em especial em Mato Grosso, Mato Grosso do Sul, Goiás, Rondônia, Bahia e mais recentemente no Sul do Maranhão. Nestes estados em que a soja está em expansão, os cultivos são extensivos, com áreas, na maioria dos casos, superiores a 1.000 hectares, chegando em alguns poucos casos com áreas que excedem a $20 \mathrm{mil}$ hectares. Tendo em vista que a rentabilidade dos grãos em geral é relativamente baixa por hectare, a sobrevivência dos produtores de soja advém de cultivos extensivos, ou seja, o lucro provém do tamanho, ou seja, da economia de escala. vras, adotar estratégias de comercialização que resultem em melhores e mais estáveis preços de mercado parece ser uma constante preocupação dos sojicultores. O presente estudo tem a intenção de analisar estratégias de comercialização de soja, levando em consideração as condições de risco de mercado.

Entre o período de plantio e a venda efetiva do produto, há geralmente um considerável risco de preços, o que ocorre notadamente com produtos agrícolas, devido aos vários fatores que afetam adversamente a produção. As flutuações dos preços da soja (volatilidade) trazem anualmente sérias preocupações aos produtores, devido à incerteza quanto a um justo retorno pelos seus esforços. A variabilidade de preços desse produto, que absorve substancial parcela do crédito rural, gera intranqüilidade também para os agentes financeiros, devido a incertezas de que o produtor obtenha receita suficiente para saldar seus compromissos bancários, caso ele não comercialize seu produto na hora certa. Isto gera, em conseqüência, maior número de inadimplências.

Além das preocupações dos produtores e dos bancos, os órgãos públicos também são afetados, direta e negativamente, em suas receitas orçamentárias, devido às inapropriadas decisões do momento correto da venda da soja, a qual contribui com ponderável participação na arrecadação paranaense.

Mesmo com a presença desta aleatoriedade nos preços da soja, é possível detectar um conjunto de estratégias ou maneiras de comercializar esse produto que possibilite uma sensível redução de risco de mercado,

O fortalecimento da maioria das cooperativas no Sul baseia-se na soja, como também é importante para o país por causa do expressivo valor gerado de divisas, via exportações. Em condições normais, o Brasil exporta quase US\$ 10 bilhões via complexo soja (grãos, farelo e óleo).

Cabe destacar que, uma vez colhida a soja, o produtor já não mais pode alterar nem a quantidade produzida e nem os custos médios de produção. Considerando-se que a equação do lucro total (L) pode ser expressa por:

$$
\mathrm{L}=\mathrm{Q}(\mathrm{Pq}-\mathrm{CMe})
$$

Onde: $\mathbf{Q}=$ quantidade produzida, $\mathbf{P q}=$ preço de mercado do produto agrícola e $\mathbf{C M e}=$ custos totais médios de produção.

Então, o lucro fica dependendo apenas do(s) preço(s) que o produtor vai conseguir ao vender seu produto. Isso significa dizer que o preço de mercado (Pq) assume importância vital na rentabilidade da atividade agrícola, principalmente após a colheita, uma vez que até os custos variáveis passam a ser fixos e o volume produzido já é dado. Em outras pala- sem afetar o nível de renda da empresa agrícola.

Devido a esse problema de flutuação de preços (risco de mercado), é de interesse dos produtores, cooperativas, bancos e firmas ligadas ao setor, avaliar as alternativas disponíveis para a comercialização de um produto (soja), porque uma eficiente seleção de opções de comercialização garantirá mais facilmente ao produtor uma receita maior e mais estável. No presente estudo, avaliam-se vinte maneiras diferentes com as quais o produtor de soja e suas cooperativas podem orientar suas decisões de "quando" e "como" vender esse produto. Para isso, são consideradas apenas as alternativas de comercialização no mercado disponível (ou físico), muito embora se saiba que os produtores também têm opções de venda da soja no mercado a termo (futuro), através da operação de "hedging". Podem também fazê-lo no mercado de opções, pela aquisição de um respectivo contrato. O hedging é uma excelente opção, mas muito pouco utilizada no Brasil, ao contrário dos Estados Unidos, em que o produtor norte-americano usa corriqueiramente vendas no mercado futuro. 


\section{METODOLOGIA}

\subsection{Alternativas de comercialização}

Como alternativa ou opção de comercialização entendese um mecanismo ou método através do qual o produtor pode vender ou influenciar os termos de venda de seu produto.

O presente estudo, conforme já referido, apresenta vinte diferentes estratégias de comercialização da soja no modelo de programação, o qual assume que todos os planos de comercialização são decididos na época de plantio. Estas estratégias envolvem somente o uso do mercado disponível. As vinte estratégias de comercialização consideradas neste estudo são:

a) Venda na Época da Colheita:

$\mathrm{EC}_{1}, \mathrm{EC}_{2}$ - Venda total da produção ou de parte dela na época da colheita, em março ou abril.

b) Armazenagem para Venda Futura: $\mathrm{EC}_{3} ; \ldots ; \mathrm{EC}_{10}$ - Armazenar a produção na época da colheita, para posterior venda futura quando o preço for compensador durante o ano.

\section{c) Vendas Diversificadas:}

$\mathrm{EC}_{11}$ - Armazenar na época da colheita, para posterior venda de $1 / 2$ em maio e $1 / 2$ em setembro.

$\mathbf{E C}_{12}$ - Armazenar na época da colheita, para posterior venda de $1 / 3 \mathrm{em}$ maio e $1 / 3$ em julho e $1 / 3 \mathrm{em}$ setembro.

$\mathbf{E C}_{13}$ - Armazenar na época da colheita, para posterior venda de $1 / 3 \mathrm{em}$ maio, $1 / 3 \mathrm{em}$ agosto e $1 / 3 \mathrm{em}$ outubro.

$\mathbf{E C}_{14}$ - Armazenar na época da colheita, para posterior venda de $1 / 4 \mathrm{em}$ abril, $1 / 4 \mathrm{em}$ junho, $1 / 4$ em agosto e $1 / 4$ em outubro.

EC $_{15}-$ Armazenar na época da colheita, para posterior venda de $1 / 2$ em agosto e $1 / 2$ em outubro.

$\mathbf{E C}_{16}$ - Armazenar na época da colheita, para posterior venda de $1 / 2$ em julho e $1 / 2$ em setembro.

$\mathbf{E C}_{17}$ - Armazenar na época da colheita, para posterior venda de $1 / 2$ em setembro e $1 / 2$ em outubro.

EC $_{18}$ - Armazenar na época da colheita, para posterior venda de $1 / 2$ em outubro e $1 / 2$ em novembro.

$\mathbf{E C}_{19}$ - Armazenar na época da colheita, para posterior venda de $1 / 2$ em setembro e $1 / 2$ em dezembro.

$\mathbf{E C}_{20}$ - Armazenar na época da colheita, para posterior venda de $1 / 3$ em abril, $1 / 3$ em setembro e $1 / 3$ em dezembro.

\subsection{A Tomada de Decisão sob Condições de Risco de Mercado}

As pressuposições da teoria da firma, de que os produ- tores têm perfeito conhecimento do valor das variáveis que afetam os processos de suas atividades e tomem decisões objetivando a maximização do lucro, têm recebido severas críticas. Essas pressuposições implicam a não-estocasticidade das variáveis, e que o único critério para a seleção de uma alternativa é o seu retorno esperado (modelo determinístico). A pressuposição da maximização do lucro, a qual implica que os empresários igualem a receita marginal ao custo marginal, é válida apenas sob condições de neutralidade de risco. Contudo, dado o ambiente de risco de produção e de mercado em que os agricultores operam, o critério da teoria clássica não auxilia muito as decisões do produtor. Conseqüentemente, isto sugere o uso de modelos alternativos (modelos estocásticos) que, explicitamente, incorporem o risco. 
de Taylor. Eles observam que isto é verdadeiro se as derivadas, além da segunda, são suficientemente pequenas para serem ignoradas ou, em outras palavras, se o risco agregado assumido pelos produtores é pequeno em proporção às riquezas totais.

Uma pressuposição comum é que a função de utilidade é estritamente côncava, o que implica que o fazendeiro é avesso ao risco. Como Francis (1976) sugere, esta é uma pressuposição razoável, porque a função de utilidade convexa implica afirmar que o produtor prefere o risco e isto representa uma situação anormal, pois, no mundo real, poucos tomadores de decisões demonstram tal comportamento. A situação comum parece ser de indivíduos avessos ao risco, uns mais outros menos. Para um empresário avesso ao risco, quanto maior a variância da renda esperada, devido a uma maior variabilidade nos preços do produto, menor o nível de utilidade deste indivíduo. Em outras palavras, a aversão ao risco implica que o agricultor requer aumentos de renda a taxas crescentes para cada unidade adicional de risco.

\subsection{O Modelo Matemático de Markowitz-Tobin}

Com base nas pressuposições de que:

a) A função de utilidade do agricultor é quadrática;

b) A distribuição de freqüência dos retornos das estratégias é normal;

c) O modelo de programação quadrática foi selecionado para avaliar as estratégias de comercialização e estimar a fronteira eficiente $(\mathrm{E}-\mathrm{V})$ de renda-risco.

A programação quadrática (PQ) é uma extensão da programação linear (PL) porque a $\mathrm{PQ}$ considera o risco, enquanto a PL o ignora. O modelo de PQ assume que: 1) o objetivo do produtor de maximização do lucro é diretamente afetado pela sua aversão ao risco; 2) à medida que o valor do coeficiente de aversão ao risco diminui, a renda esperada aumenta; 3) o aumento na renda está associado a uma maior variabilidade da mesma.

Segundo Markowitz (1959), Takayama e Batterham (1972) e Mendes (1980), a derivação do modelo matemático de Markowitz-Tobin que vai ser utilizado neste estudo para a seleção de portfólios eficientes será discutida a seguir.

Inicialmente, definem-se as variáveis:

$\mathrm{p}_{\mathrm{ij}}=$ Preço médio real esperado por unidade de atividade $\mathrm{i}$ no mês j $(i=1,2 . . n ; j=1, \ldots, 12)$;

$\sigma_{i j}=$ Covariância do preço médio real por unitário da atividade i no mês j;

$\mathrm{X}_{\mathrm{ij}}=$ Unidades de produção esperadas alocadas para a atividade i no mês j;
$\mathrm{A}_{\mathrm{ij}}=$ Custo de armazenagem por unidade de atividade i no mês j;

$\mathbf{T}_{\mathrm{ij}}=$ Custo de transporte por unidade de atividade i no mês j;

$\mathbf{R}_{\mathrm{ij}}=$ Custo de repagamento de empréstimo (crédito rural) por unidade de atividade i no mês j;

$\mathbf{C}=$ Custo variável real menos a necessidade de recursos financeiros (crédito rural) por unidade de atividade $\mathrm{i}$ no mês j.

Qualquer combinação específica de $\mathrm{n}$ atividades de risco vai proporcionar um retorno esperado de:

$$
E=\sum_{i=1}^{n} \sum_{j=1}^{n} p_{i j} x_{i j}-\sum_{i=1}^{n} \sum_{j=1}^{n}\left(A_{i j}+T_{i j}+R_{i j}+C\right) \cdot x_{i j}
$$

Sendo a variância do retorno líquido esperado igual a:

$$
\mathbf{v}_{(\mathrm{E})}=\sum_{i=1}^{n} \sum_{j=1}^{12} \sigma_{i j} x_{i j} x_{i j}
$$

Substituindo as equações (1) e (2) na função de utilidade quadrática do tomador de decisão (3), o problema passa a ser representado por um conjunto de valores para $\mathrm{x}_{\mathrm{i}}$ que maximizam a equação (4):

$$
\mathrm{U}=\mathbf{E}+\mathbf{b E}^{2}+\mathbf{c V}
$$

$$
\begin{aligned}
U= & \sum_{i=1}^{n} \sum_{j=1}^{12} p_{i j} x_{i j}-\sum_{i=1}^{n} \sum_{j=1}^{12}\left(A_{i j}+T_{i j}+R_{i j}+C\right) x_{i j}+b\left(\sum_{i=1}^{n} \sum_{j=1}^{12} p_{i j} x_{i j}\right. \\
& \left.-\sum_{i=1}^{n} \sum_{j=1}^{12}\left(A_{i j}+T_{i j}+R_{i j}+C\right) x_{i j}\right)^{2}+c \sum_{i=1}^{n} \sum_{j=1}^{12} \sigma_{i j} x_{i j} x_{i j}
\end{aligned}
$$

Sujeito a um conjunto de restrições de recursos, assim como a $X_{i} \geq 0$.

Mendes (1980) cita que Taha (1976, p. 327) concluiu que, "usando a expansão pela série de Taylor, podemos demonstrar que os três primeiros termos da função de utilidade esperada proporcionam um critério semelhante ao apresentado a seguir":

$Z=\sum_{i=1}^{n} \sum_{j=1}^{12} p_{i j} x_{i j}-\sum_{i=1}^{n} \sum_{j=1}^{12}\left(A_{i j}+T_{i j}+R_{i j}+C\right) x_{i j}-\alpha \sum_{i=1}^{n} \sum_{j=1}^{12} \sigma_{i j} x_{i j} x_{i j}$

a qual, em notação matricial, poderia ser representada por:

$$
Z=P X-\left(A^{+} T+R+C\right) X^{-} \alpha X^{\prime} D X
$$

Onde:

$\mathrm{Z}=$ função objetiva a ser maximizada;

$\mathrm{P}=$ vetor-linha dos preços médios unitários por estratégia de comercialização;

$\mathrm{X}=$ vetor-coluna do nível de estratégias de comercialização da soja; 
A = vetor-linha dos custos unitários de armazenagem por estratégia/mês;

$\mathrm{T}=$ vetor-linha dos custos unitários de transporte por estratégia/mês;

$\mathrm{R}=$ vetor-linha dos custos unitários de repagamento por estratégia/mês;

$\mathrm{C}=$ escalar do custo variável de produção;

$\alpha=$ coeficiente de aversão ao risco ou escalar;

D = matriz de variância-covariância.

Taha (1976), enfatizou que a expressão (5) é compatível com o uso da utilidade como critério na decisão, pois o fator da aversão ao risco $(\alpha)$ é um indicador da atitude do empresário frente a um desvio excessivo dos valores esperados.

Portanto, o objetivo é maximizar a expressão (5) sujeita às seguintes restrições:

$$
\begin{gathered}
R V=\sum_{i=1}^{n} \sum_{j=1}^{12} x_{i j}=1 \\
\mathrm{~A} \mathrm{X} \leq \mathrm{B} \\
\mathrm{X} \geq 0
\end{gathered}
$$

Onde:

$\mathrm{RV}=$ restrição de venda;

$\mathrm{A}=$ coeficiente da matriz de insumo-produto; $\mathrm{e}$

$\mathrm{B}=$ vetor coluna dos níveis de recursos e outras restrições.

De acordo com Takayama e Batterham (1972), neste modelo, $\alpha$ desempenha o papel de ajustar o ótimo das estratégias de comercialização. Desta forma o escalar $\alpha$, aplicado às relações de variância-covariância para refletir a aversão ao risco, sofre parametrização entre o intervalo de 0 e 1 , em valor absoluto, objetivando gerar soluções para pessoas com comportamento diferente em relação ao risco de mercado. $\mathrm{O}$ valor zero para o coeficiente de aversão ao risco $(\alpha=0)$, ou seja, a não existência do elemento risco, vai converter o modelo de PQ em um modelo de PL (produtores indiferentes frente ao risco de mercado). À medida que a parametrização aumenta, ou seja, $\alpha$ sofre incremento, o modelo fornece planos de comercialização formados por um conjunto de estratégias de comercialização.

\section{RESULTADOS E DISCUSSÃO}

O modelo de programação quadrática, pela maximização da função objetivo, especificada na equação 5 , proporciona um plano ótimo (portfólio), o qual compreende uma es- tratégia ou a combinação de um conjunto de estratégias de comercialização da produção de soja.

Segundo Takayama e Batterham (1972), conforme varia o coeficiente de aversão ao risco $(0<\alpha<1)$, uma série de planos ótimos é gerada, uma vez que há um plano ótimo para cada escalar. Altos valores para o coeficiente de aversão ao risco vão implicar, necessariamente, um alto grau de aversão ao risco por parte dos produtores rurais. De outra maneira, se o coeficiente de aversão ao risco aproximar-se de zero, o produtor tenderá a permanecer indiferente ao risco.

Quando o elemento risco é nulo, ou seja, se o coeficiente de aversão ao risco é zero ou se a matriz de variânciacovariância é uma matriz nula, então, o plano ótimo de comercialização de soja obtido é o mesmo proporcionado pelo modelo de programação linear. Neste estudo, dado que a matriz de variância-covariância não é uma matriz nula, então, o completo desconhecimento do risco só é possível no caso em que o coeficiente de aversão ao risco for igual a zero.

Os resultados obtidos pelo Modelo demonstram que produtores de soja com "maior aversão ao risco" selecionam, a princípio, um conjunto de estratégias de comercialização diferente daquele selecionado por tomadores de decisão que possuem uma "menor aversão ao risco".

O plano ótimo mais eficiente dentre todos, ou seja, a melhor combinação de Estratégias de Comercialização da produção proporcionada pelo Modelo diz respeito aos produtores com "média aversão ao risco" $(\alpha=\mathbf{0 , 3 0})$, porque em relação à Margem Bruta Esperada (MBE) da situação de neutralidade de risco, haveria uma queda de $11,6 \%$ na mesma, e uma redução do risco em torno de $95,2 \%$.

\section{A versão ao risco implica que o agricultor Irequer aumentos de renda a taxas crescentes para cada unidade adicional de risco.}

De forma comparativa, os produtores ditos "indiferentes ao risco", ou seja, aqueles que estão dispostos a assumir grandes riscos na comercialização de sua produção escolheriam $\alpha=\mathbf{0 , 0}$ (zero), obtendo, assim, uma Margem Bruta Esperada máxima, que coincide com a solução fornecida pela programação linear.

Desse modo, no plano ótimo mais eficiente $(\alpha=\mathbf{0 , 3 0})$, as estratégias de comercialização eram as seguintes: venda de $1,8 \%$ da produção através da estratégia $\mathrm{EC}_{6}$ (agosto), $7,6 \%$ através da estratégia $\mathrm{EC}_{7}$ (venda em setembro), 10,5\% através da estratégia $\mathrm{EC}_{8}$ (venda em outubro), 13,7\% através da estratégia $\mathrm{EC}_{9}$ (venda em novembro), $15,7 \%$ através da estratégia $\mathrm{EC}_{10}$ (venda em dezembro), 7,0\% através da 
estratégia $\mathrm{EC}_{15}$ (venda de $1 / 2$ em agosto e $1 / 2$ em outubro), $2,9 \%$ através da estratégia $\mathrm{EC}_{16}$ (venda de $1 / 2$ em julho e $1 / 2$ em setembro), 9,3\% através da estratégia $\mathrm{EC}_{17}$ (venda de $1 / 2$ em setembro e $1 / 2$ em outubro), $12,1 \%$ através da estratégia $\mathrm{EC}_{18}$ (venda de $1 / 2$ em outubro e $1 / 2$ em novembro), $11,8 \%$ através da estratégia $\mathrm{EC}_{19}$ (venda de $1 / 2$ em setembro e $1 / 2 \mathrm{em}$ dezembro) e 6,5\% através da estratégia $\mathrm{EC}_{20}$ (venda de $1 / 3$ em abril, $1 / 3$ em setembro e $1 / 3$ em dezembro), conforme se pode observar na tabela 1 .

\section{comportamento dos produtores de soja
varia significativamente conforme se altera} o coeficiente de aversão ao risco.

Tal plano ótimo de comercialização corresponde à armazenagem da produção na época da colheita com posterior venda mensal fracionada pela seguinte composição: $2,2 \%$ em abril, $1,5 \%$ em julho, 5,5\% em agosto, $21,9 \%$ em setembro, $25,1 \%$ em outubro, $19,9 \%$ em novembro e $23,9 \%$ em dezembro, em que $90,2 \%$ de todas as vendas se concentrariam nos últimos quatro meses do ano. Essa estratégia proporciona uma margem bruta esperada de $\mathrm{R} \$ 15,34$ por saca e um risco (possibilidade de perda devido ao desvio-padrão em relação à $\mathrm{MBE}$ ) de $\mathrm{R} \$ 1,38$ por saca (vide Tabela 1 e 2 ).

Para produtores com "elevada aversão ao risco" $(\alpha=\mathbf{1 , 0})$, o Modelo de programação quadrática selecionou uma combinação ótima de vendas com uma composição diferente da solução obtida anteriormente (produtores com "média aversão ao risco"), diversificando bastante as vendas com objetivo de reduzir o risco de mercado associado a esta atividade de venda.

Assim sendo, tem-se a comercialização de 3,4\% da sua produção na época da colheita em março; $4,8 \%$ em abril; 5,6\% em maio; $3,9 \%$ em junho; 7,5\% em julho; $10,2 \%$ em agosto; $20,7 \%$ em setembro; $17,9 \%$ em outubro; $10,9 \%$ em novembro e $15,2 \%$ em dezembro.

As estratégias selecionadas para esse nível de risco foram as seguintes: $3,4 \%$ através da estratégia $\mathrm{EC}_{1} ; 0,8 \%$ através da estratégia $\mathrm{EC}_{2} ; 2,5 \%$ através da estratégia $\mathrm{EC}_{4} ; 3,6 \%$ através da estratégia $\mathrm{EC}_{5} ; 4,4 \%$ através da estratégia $\mathrm{EC}_{6} ; 5,3 \%$ através da estratégia $\mathrm{EC}_{7} ; 5,9 \%$ através da estratégia $\mathrm{EC}_{8} ; 7,5 \%$ através da estratégia $\mathrm{EC}_{9} ; 8,9 \%$ através da estratégia $\mathrm{EC}_{10}$ e 8,0\% através da estratégia $\mathrm{EC}_{20}$. Então, pode-se perceber que o modelo utilizou 19 das 20 estratégias existentes para formar um portfólio eficiente com o menor nível de risco possível para esta categoria de produtores.

Essa estratégia proporciona uma margem bruta esperada de $\mathrm{R} \$ 13,55$ por saca e um risco (possibilidade de perda devi- do ao desvio-padrão em relação à $\mathrm{MBE}$ ) de $\mathrm{R} \$ 1,12$ por saca, sendo considerada como a estratégia mais conservadora dentre todas as calculadas, pela baixa aceitação de risco por parte do tomador de decisão.

Analisando-se, ainda, a Tabela 1, observa-se que a estratégia EC9 participou praticamente de todos os planos ótimos de comercialização, demonstrando, além do maior valor esperado para a Margem Bruta, a importância da introdução do conceito de covariâncias na seleção de um conjunto de estratégias de comercialização, para a composição de um plano ótimo (portfólio).

A importância das covariâncias na análise baseia-se no conceito de diversificação, desenvolvido originalmente por Markowitz, no qual combinações de estratégias com correlação positiva menos que perfeita (covariância negativa) podem reduzir o risco de um plano ótimo sem acarretar com isto uma redução drástica na renda média esperada dos produtores.

Em virtude disso, uma estratégia de comercialização adiciona mais ou menos variabilidade ao plano ótimo dependendo da soma de suas covariâncias com as covariâncias de outras estratégias.

Quando a matriz de variância-covariância gerada não possuir elementos com covariâncias negativas, o modelo é forçado a utilizar-se das covariâncias positivas na seleção de estratégias para a composição de um plano ótimo. O problema, nesse caso, consiste no fato de que duas estratégias com covariâncias positivas resultam em um aumento de variabilidade e risco a um plano ótimo.

O grau de aversão ao risco por parte do produtor afeta diretamente a combinação de estratégias de comercialização dos planos ótimos (Tabela 1). Sendo assim, produtores com "alto grau de aversão ao risco" selecionarão estratégias diversificadas de vendas que proporcionam uma menor Margem Bruta Esperada, com o objetivo básico de reduzir o risco de seu plano ótimo de vendas.

Por outro lado, produtores com "baixa aversão ao risco", ou seja, os que objetivam maximização vão selecionar estratégias que normalmente concentrem suas vendas e que proporcionem uma elevada Margem Bruta Esperada, mesmo que com isto resulte um plano ótimo de vendas com alto risco associado.

\subsection{A Fronteira Eficiente (Fronteira E-STD)}

A fronteira eficiente é um aspecto importante a ser considerado na análise de planos ótimos de comercialização (portfólios). Dessa forma, as informações relevantes sobre as estratégias de comercialização podem ser resumidas em três medidas: a média do retorno esperado (tomada como a média 


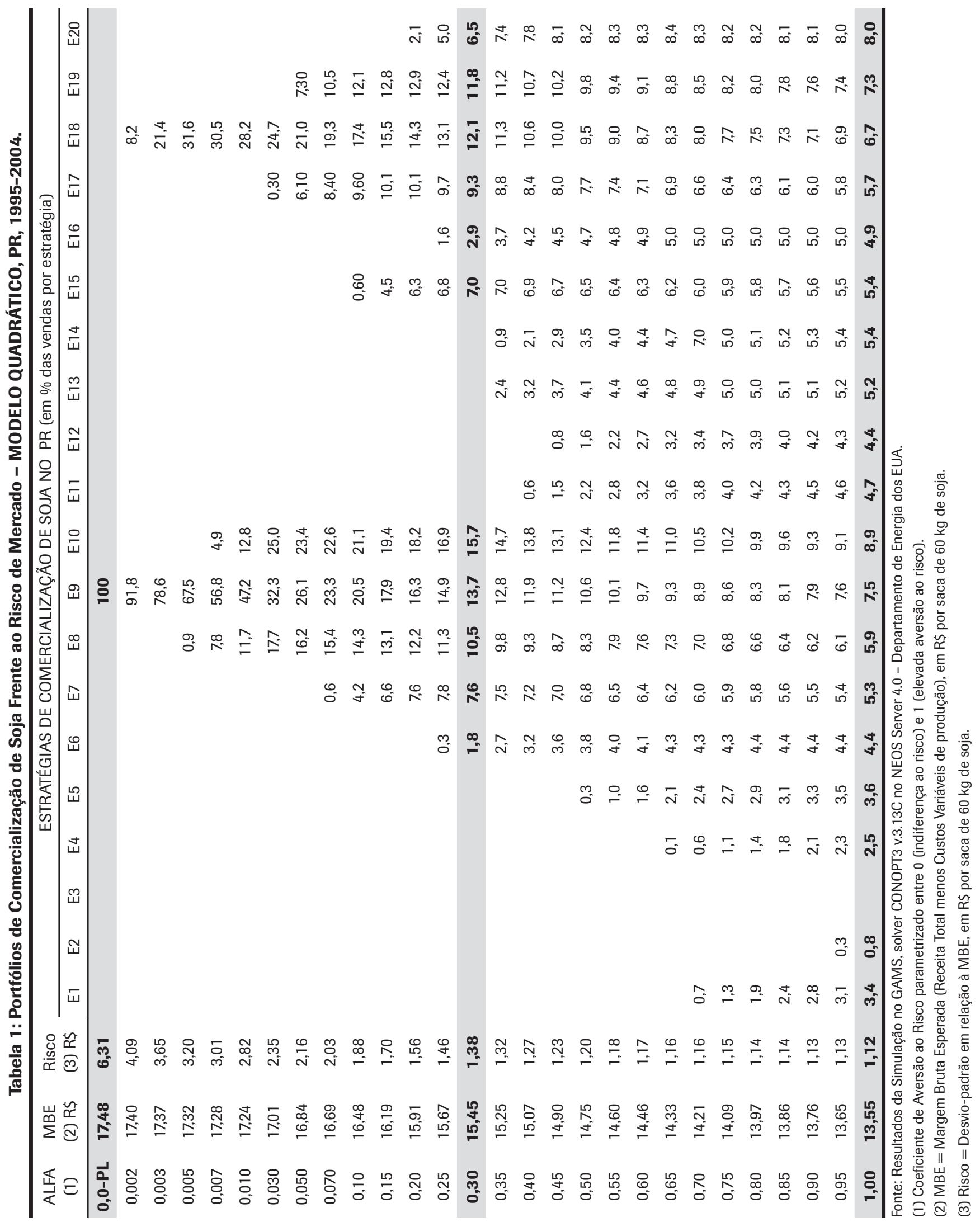


aritmética), o desvio-padrão dos retornos e a correlação com os outros retornos das demais estratégias de comercialização.

A média e o desvio-padrão podem ser usados para delinear o risco relativo e o retorno de qualquer seleção de estratégias de comercialização. A fronteira eficiente estriba-se na dominância de certos princípios, de tal forma que, entre todos os investimentos com uma dada taxa de retorno, o que oferece mínimo risco é o mais desejável; ou, entre todas as alternativas de uma dada classe de risco, a que oferece a mais alta taxa de retorno é a preferível.

Como resultado, pontos sobre a fronteira compreendem planos ótimos de estratégias de comercialização (portfólios), pontos esses em que o produtor seleciona um setor que é capaz de maximizar sua utilidade esperada, para dado coeficiente de aversão ao risco que está disposto a assumir (Conforme se pode observar na Figura 1).

Tabela 2: Percentagem de Venda de Soja, Discriminação Mensal, segundo Coeficiente de Aversão ao Risco - Modelo Básico - PR, 1995-2004.

\begin{tabular}{|c|c|c|c|c|c|c|c|c|c|c|}
\hline \multirow{2}{*}{$\begin{array}{c}\text { Coeficiente de Aversão } \\
\text { ao Risco ( ) }\end{array}$} & \multicolumn{10}{|c|}{ Discriminação Mensal de Vendas dos Planos Ótimos (\%) } \\
\hline & MAR. & ABR. & MAI. & JUN. & JUL. & AGO. & SET. & OUT. & NOV. & DEZ. \\
\hline 0,0 (P.L.*) & - & - & - & - & - & - & - & - & 100,0 & - \\
\hline 0,002 & - & - & - & - & - & - & - & 4,1 & 95,9 & - \\
\hline 0,003 & - & - & - & - & - & - & - & 10,7 & 89,3 & - \\
\hline 0,005 & - & - & - & - & - & - & - & 16,7 & 83,3 & - \\
\hline 0,007 & - & - & - & - & - & - & - & 23,1 & 72,1 & 4,9 \\
\hline 0,010 & - & - & - & - & - & - & - & 25,8 & 61,3 & 12,9 \\
\hline 0,030 & - & - & - & - & - & - & 0,2 & 30,2 & 44,7 & 25,0 \\
\hline 0,050 & - & - & - & - & - & - & 6,7 & 29,8 & 36,6 & 27,0 \\
\hline 0,070 & - & - & - & - & - & - & 10,0 & 29,3 & 33,0 & 27,8 \\
\hline 0,10 & - & - & - & - & - & 0,4 & 15,1 & 28,2 & 29,2 & 27,2 \\
\hline 0,15 & - & - & - & - & - & 2,3 & 18,1 & 28,2 & 25,7 & 25,8 \\
\hline 0,20 & - & 0,7 & - & - & - & 3,2 & 19,8 & 27,6 & 23,5 & 25,4 \\
\hline 0,25 & - & 1,7 & - & - & 0,8 & 3,7 & 21,4 & 26,1 & 21,5 & 24,8 \\
\hline 0,30 & - & 2,2 & - & - & 1,5 & 5,5 & 21,9 & 25,1 & 19,9 & 23,9 \\
\hline 0,35 & - & 2,7 & 0,8 & 0,2 & 1,9 & 7,2 & 21,7 & 24,4 & 18,5 & 22,7 \\
\hline 0,40 & - & 3,1 & 1,4 & 0,5 & 2,1 & 8,2 & 21,8 & 23,9 & 17,3 & 21,8 \\
\hline 0,45 & - & 3,4 & 2,3 & 0,7 & 2,5 & 8,9 & 22,1 & 23,0 & 16,2 & 20,9 \\
\hline 0,50 & - & 3,6 & 3,0 & 0,9 & 3,2 & 9,3 & 22,3 & 22,4 & 15,4 & 20,0 \\
\hline 0,55 & - & 3,8 & 3,6 & 1,0 & 4,1 & 9,7 & 22,2 & 21,8 & 14,6 & 19,3 \\
\hline 0,60 & - & 3,9 & 4,0 & 1,1 & 5,0 & 9,9 & 22,2 & 21,2 & 14,0 & 18,7 \\
\hline 0,65 & - & 4,0 & 4,5 & 1,3 & 5,7 & 10,2 & 22,2 & 20,7 & 13,5 & 18,2 \\
\hline 0,70 & 0,7 & 4,1 & 4,7 & 1,9 & 6,0 & 10,2 & 21,9 & 20,2 & 12,8 & 17,6 \\
\hline 0,75 & 1,3 & 4,0 & 4,9 & 2,4 & 6,4 & 10,2 & 21,7 & 19,7 & 12,5 & 17,0 \\
\hline 0,80 & 1,9 & 4,0 & 5,0 & 2,7 & 6,7 & 10,2 & 21,5 & 19,3 & 12,1 & 16,6 \\
\hline 0,85 & 2,4 & 4,0 & 5,2 & 3,1 & 6,9 & 10,3 & 21,2 & 19,0 & 11,8 & 16,2 \\
\hline 0,90 & 2,8 & 4,0 & 5,4 & 3,4 & 7,2 & 10,2 & 21,2 & 18,6 & 11,5 & 15,8 \\
\hline 0,95 & 3,1 & 4,3 & 5,5 & 3,7 & 7,4 & 10,2 & 21,0 & 18,3 & 11,1 & 15,5 \\
\hline 1,00 & 3,4 & 4,8 & 5,6 & 3,9 & 7,5 & 10,2 & 20,7 & 17,9 & 10,9 & 15,2 \\
\hline
\end{tabular}

Fonte: Resultados do modelo básico, (P.L.) = solução obtida por programação linear. 
Analisando-se a Figura 1, é possível observar que a inclinação da fronteira eficiente tende a decrescer enquanto a Margem Bruta Esperada sofre incremento. Este aumento no risco (desvio-padrão) ocorre devido ao fato de que, com o incremento na Margem Bruta, a seleção das estratégias de comercialização para a composição do plano ótimo sofre restrições para que se possa obter o nível de Margem Bruta desejado, proporcionando, então, uma redução na diversificação das estratégias.

O ponto $\mathbf{P}$ na Figura 1 representa o plano ótimo de comercialização do produtor "indiferente ao risco", que é proporcionado pela programação linear, cuja Margem Bruta Esperada é máxima ( $\mathrm{R} \$ 17,48$ por saca), mas com um risco associado (desviopadrão) muito elevado ( $\mathrm{R} \$ 6,31$ por saca), sendo descartado como o ideal.

O ponto A, por outro lado, representa uma situação completamente oposta, indicando o produtor "altamente avesso ao risco", cuja Margem Bruta Esperada ( $\mathrm{R} \$ 13,55$ por saca) possui o menor risco associado dentre todos ( $\mathrm{R} \$$ 1,12 por saca), mas que também não é a solução ideal de comercialização.

$\mathrm{O}$ ponto $\mathbf{B}$, diferentemente dos anteriores, pode ser considerado a solução ideal proporcionada pelo modelo de programação quadrática, pois, além de ser o ponto de tangência da curva de isoutilidade, tem, na sua diversificação de vendas, uma motivação para a redução de risco (R\$ 1,38 por saca) ao plano ótimo de $95,2 \%$. Por outro lado, conseguiu manter uma Margem Bruta Esperada (R\$ $15,45) 11,6 \%$ inferior à obtida pelo ponto $\mathrm{P}$ (programação linear).

\section{$\Delta$ fronteira eficiente é um aspecto importante a Aser considerado na análise de planos ótimos de comercialização (portfólios).}

Os resultados analisados anteriormente confirmam esta afirmação, dado que, em planos ótimos com "alta aversão ao risco", são constituídos por mais de 10 estratégias de comercialização, enquanto que em planos ótimos com "indiferença ao risco", é composto por somente uma estratégia de comercialização, o que consiste, basicamente, no ponto de maximização de lucro fornecido pela programação linear.

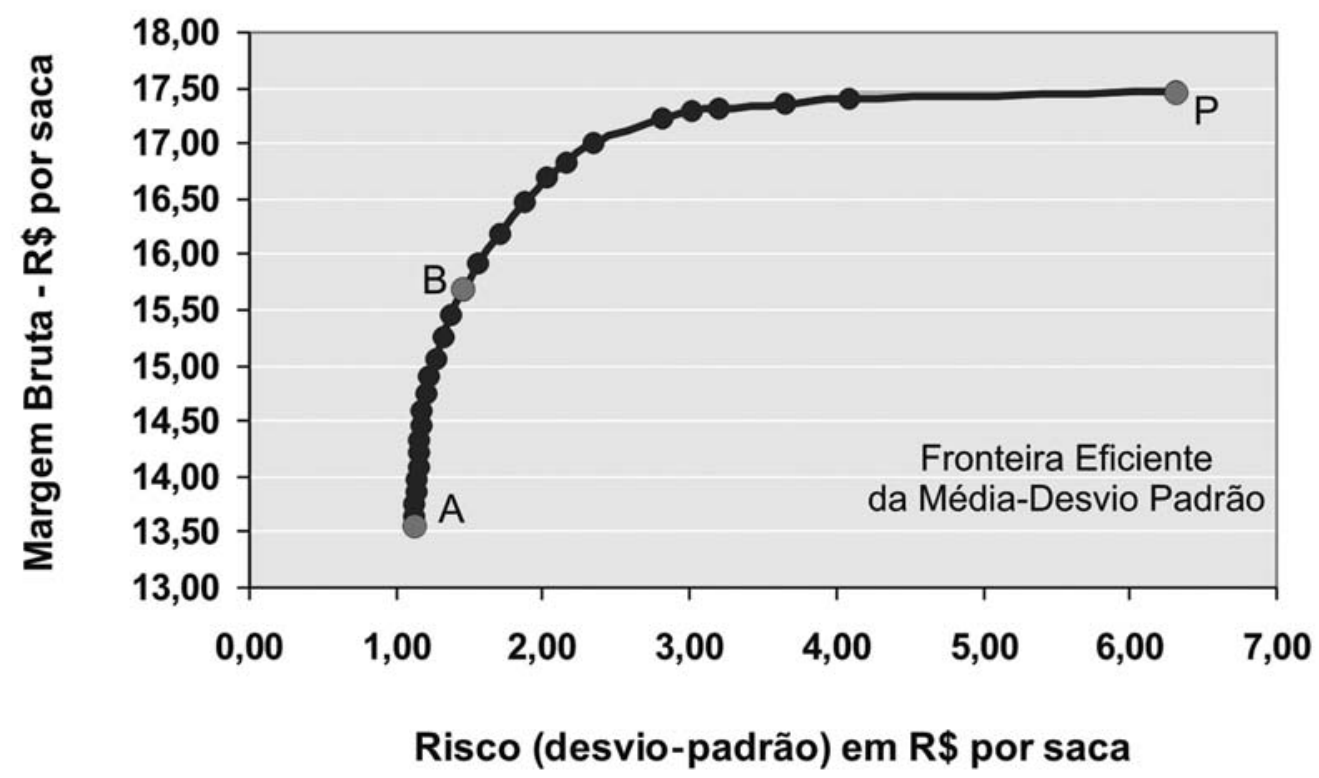

Figura 1: A Fronteira Eficiente para as Estratégias de Comercialização de Soja - PR, 1995-2004.

Fonte: Resultados obtidos pelo Modelo Básico. 


\section{CONCLUSÕES}

Os resultados fornecidos pelo Modelo analisado deixam claro que o comportamento dos produtores de soja varia significativamente conforme se altera o coeficiente de aversão ao risco, resultando em conjuntos diferentes de estratégias de comercialização para a formação de seu plano ótimo de vendas.

A melhor combinação de estratégias de comercialização de soja frente ao risco de mercado para o estado do Paraná, no período de 1995-2004 (ou seja, o ponto de tangência entre a Fronteira Eficiente da Média-Desvio padrão e as Curvas de Isoutildade dos produtores) constitui-se na seleção realizada por produtores com "média aversão ao risco" $(\alpha=0,3)$.

Tal situação pode ser explicada pela combinação diversificada de vendas implicar uma redução de 95,2\% no risco associado ao plano ótimo de vendas, conseguindo, ainda, manter uma Margem Bruta Esperada apenas 11,6\% inferior à solução obtida pela programação linear.

Assim sendo, a maneira mais apropriada de comercializar a soja, tendo em vista a redução de risco e da variabilidade na comercialização, seria através da armazenagem da produção na época da colheita e a venda em lotes parcelados no mercado disponível através da seguinte composição mensal de vendas: $2,2 \%$ em abril, $1,5 \% \mathrm{em}$ julho, 5,5\% em agosto, $21,9 \%$ em setembro, 19,9\% em novembro e $23,9 \%$ em dezembro, onde $90,8 \%$ das vendas totais estariam concentradas nos últimos quatro meses do ano (vide Tabela 3 ).

Finalmente, deve-se enfatizar que os resultados obtidos neste estudo possuem certas limitações oriundas tanto do modelo utilizado quanto das hipóteses simplificadoras que foram utilizadas para representar a realidade e que são válidas exclusivamente para a região analisada.

Assim, é necessário evitar generalizações que possam ser derivadas da presente análise empírica, pois ela serviu apenas para ilustrar o fato de que existem várias alternativas e ferramentais para o planejamento de comercialização.
Para estudos futuros, propõem-se a atualização deste trabalho através da utilização da Dominância Estocástica, que vem a ser um ferramental semelhante ao da análise E-STD, e a comparação dos resultados para a determinação da eficiência de ambos diante de situações de tomada de decisões frente ao risco de mercado.

Tabela 3: Discriminação Percentual Mensal das Vendas de Soja Segundo o Coeficiente de Aversão ao Risco $\alpha=0,3$, PR - 1995-2004.

\begin{tabular}{cc}
\hline Meses de Venda da Soja & $\begin{array}{c}\text { Discriminação \% Mensal de } \\
\text { Comercialização da soja }\end{array}$ \\
\hline março & - \\
abril & 2,2 \\
maio & - \\
junho & - \\
julho & 1,5 \\
agosto & 5,5 \\
setembro & 21,9 \\
outubro & 25,1 \\
novembro & 19,9 \\
dezembro & 23,9 \\
\hline
\end{tabular}

Fonte: Resultados obtidos pelo Modelo Básico.

\section{NOTAS}

1. O termo "plano" é aqui definido como uma das vinte estratégias de comercialização ou, o que é mais comum, uma combinação delas (portfólio).

2. Como Anderson et al. (1977) concluem, "the neoclassical theory of the firm with its assumptions or certainty and linear utility is inadequate for normative analysis of risk production where preference for profits is nonlinear. An approach involving decision anlaysis methods is clearly more appropriate". 


\section{REFERÊNCIAS}

MARKOWITZ, H. Portfolio Selection: efficiente diversification of investments. New York: John Wiley, 1959.

MENDES, J. T. G. The selection of marketing strategies under price risk: the case of brasilian soybeans. Columbus, Ohio, The Ohio State University, (Ph.D. thesis), 1980.

TAKAYAMA, T. \& BATTERHAM, R. L. Portfolio Selection and Resource Allocation for Financial and Agricultural Firms With the Rand QP360 Quadratic Programming Code. Ilinois, DAE -
University of Ilinois at Urbana-Champaign, 19 p. il., 1972.

TAHA, H. A. Operations Research: An Introduction, Second Edition, MacMillan Publishing Co., Inc., New York, 1976.

\section{SOBRE OS AUTORES}

\section{Judas Tadeu Grassi Mendes}

Estação Business School - IBMEC

End.: Av. Sete de Setembro, 2775, 8o andar - Rebouças - CEP 80.230-010 - Curitiba - Paraná

Tel.: (41) 2101-8800

E-mail: Judas@estacaopr.com.br

\section{João Batista Padilha Junior}

Departamento de Economia Rural e Extensão

Universidade Federal do Paraná

End.: Rua dos Funcionários, 1540 - Juvevê - CEP: 80.035-050 - Curitiba - Paraná.

Tel./Fax: (41) 3350-5604

E-mail: jpadilha@ufpr.br 